\title{
INCORPORACIÓN DE LAS TIC EN LA EDUCACIÓN. RECOMENDACIONES DE ORGANISMOS DE COOPERACIÓN INTERNACIONAL 1972-2018
}

\author{
Incorporation of ICT in Education.
} Recommendations of International Cooperation Agencies 1972-2018

Carlos Enrique George-Reyes ${ }^{1}$

cgeorgemx@gmail.com
${ }^{1}$ Universidad de las Américas Puebla, México

Fecha de recepción: 4/05/2020

Fecha de aprobación: 20/06/2020

\section{Resumen}

La incorporación de las tecnologías de la información y la comunicación (TIC) en la educación superior surge como resultado del discurso de organismos de cooperación internacional que, por sus atribuciones, tienen la capacidad de emitir recomendaciones de alcance internacional que posicionan a las TIC como herramientas estratégicas para lograr la transformación de la Educación Superior. En esta investigación se realiza el análisis de las recomendaciones de tres organismos, la Unesco, la OCDE y la OEI, con el objetivo de reconocer su posicionamiento sobre el tema a lo largo de las últimas décadas. Los resultados indican que estos organismos utilizan sus voces para influir en el diseño de las políticas de Estado al justificar la incorporación de las tecnologías en la educación como un sinónimo de la mejora del proceso educativo mediante el uso de dispositivos digitales en la práctica docente, sin embargo, se concluye que asumir de forma acrítica dichas voces puede representar un riesgo si no se considera la heterogeneidad de las capacidades de infraestructura y necesidades académicas de las universidades, así como de las habilidades de profesores y alumnos con el uso de herramientas digitales.

Palabras clave: docencia, educación, organización internacional, política educacional, tecnología educacional, TIC.

\section{Abstract}

The integration of Information and Communication Technologies (ICT) in Higher Education arises through the discourse of international cooperation agencies capable of issuing global recommendations that position ICT as strategic tools to achieve the transformation of Education Higher. This research makes an analysis of the recommendations of three organizations, Unesco, the OECD and the OEI with the aim of recognizing their position on the subject over the last decades. The results indicate that these organizations use their voices to influence the design of state policies by justifying the incorporation of technologies in education as a synonym for the improvement of the educational process through the use of digital devices in teaching practice, without However, it is concluded that assuming such voices uncritically can represent a risk if the heterogeneity of infrastructure capacities and academic needs in educational institutions is not considered, as well as the skills of teachers and students with the use of digital tools.

Keywords: teaching, education, international organization, educational policy, educational technology, ICT. 


\section{Revista Caribeña de Investigación Educativa | 2021, 5(1), 101-115}

\section{Introducción}

En los últimos años, el modelo económico-social imperante en el mundo es el capitalista (salvo contadas excepciones), y actualmente se encuentra en una fase de globalización que abarca las esferas económica, educativa y cultural. Esto ha provocado un cambio radical de la gestión, uso y dominio de las tecnologías, especialmente en el campo de la educación (Lázaro et al., 2018; Fuentes et al., 2019). En este escenario, los organismos de cooperación internacional han definido de forma coherente la importancia de hacer omnipresentes las tecnologías de la información y la comunicación (TIC) en las instituciones educativas (Arellano, 2014; Giraldo \& Báquiro, 2020); en consecuencia, los gobiernos de todo el mundo han diseñado políticas de Estado orientadas a fortalecer la incorporación de estas herramientas en los sistemas educativos, ya que son consideradas como estratégicas para el desarrollo de una nación, entre otras razones, porque se tiene la firme idea de que pueden producir nuevas formas de enseñanza que permitan alcanzar altos indicadores internacionales de eficacia y eficiencia educativa.

La intención del presente artículo es proporcionar elementos para reflexionar acerca de la forma en que tres distintos organismos de cooperación internacional influyen con sus recomendaciones en las políticas de Estado, por lo que, utilizando como estrategia metodológica la investigación documental, se analizaron diversos documentos publicados entre 1972 y 2018 que en sus líneas han plasmado la idea de que incorporar las TIC en la educación superior puede generar un cambio efectivo en la enseńanza y trasformar la práctica docente.

El análisis realizado permitió revisar la evolución de las principales premisas en el tema de tres organismos internacionales de gran influencia en el mundo: la Organización de las Naciones Unidas para la Educación, la Ciencia y la Cultura (Unesco), la Organización para la Cooperación y el Desarrollo Económicos (OCDE) y la Organización de Estados Iberoamericanos (OEI), así como su relación con las políticas TIC implementadas por algunos Estados latinoamericanos.

\section{Consideraciones}

Teun van Dijk (2009) afirma que en los estudios del discurso político no existen estrategias específicas para hacer un análisis crítico. Esto se debe a que los objetivos e intereses del investigador son primordiales para formular las acciones que orientan los propios análisis. Sin embargo, sí identifica distintas formas para estudiar las estructuras de un texto. En ese sentido, se planteó como estrategia metodológica la investigación documental con un enfoque cualitativo, en la que se recopilaron documentos mediante una selección, articulación y lectura crítica de sus contenidos textuales con el fin de orientar un proceso sistemático y hermenéutico.

De esta forma, y de acuerdo con García y Ramírez (2019), primero se llevó a cabo un proceso de planeación en el que se definió que, con base en la literatura disponible de los repositorios institucionales de organismos de cooperación internacionales, se analizarían las recomendaciones de la Unesco, la OCDE, y la OEI en materia de incorporación de tecnologías en la educación superior en el lapso comprendido entre 1972 y 2018.

Posteriormente se obtuvo la información y se organizó en fichas de análisis mediante las cuales se pudieron establecer etapas, relaciones, diferencias y posturas de las recomendaciones emitidas por los organismos ya mencionados; finalmente se llevó a cabo el análisis, la interpretación y la presentación de los resultados mediante los siguientes apartados: 1) La educación y el uso de las TIC, 2) Organismos de cooperación internacional y educación con TIC, 3) Recomendaciones de organismos de cooperación internacional, 4) Implicaciones de las recomendaciones en TIC.

\section{El uso de las TIC en la educación}

Las TIC se han convertido en herramientas cercanas a las personas, en particular para los estudiantes el mundo actualmente es un escenario interconectado en el que los teléfonos móviles, las redes sociales, los 
videojuegos y el acceso a dispositivos digitales como tabletas y computadoras portátiles ofrecen la oportunidad de tener al alcance un número extenso de información y recursos digitales.

En el escenario de la educación superior, los organismos de cooperación internacional, en su calidad de entes orientadores y ordenadores de las políticas globales, han ejercido una influencia histórica para determinar el diseño de políticas públicas de sus países miembros con el fin de lograr que la interconexión tecnológica demandada por la sociedad del conocimiento pueda trasladarse a las aulas, generar procesos de aprendizaje innovadores y lograr una nueva masificación de la educación.

En respuesta a estas orientaciones, los gobiernos de los países latinoamericanos empezaron a incorporar infraestructura tecnológica en el sistema escolar en el nivel superior desde hace ya varias décadas (Cabero et al., 2020), lo que originó el surgimiento de diversas políticas educativas nacionales diseñadas para impulsar la formación de los futuros egresados a la sociedad del conocimiento y con ello hacerlos partícipes del desarrollo económico y social en sus contextos de actuación profesional (WEF, 2016).

Sin embargo, se puede afirmar que la incorporación tiene un sesgo de racionalidad económica, ya que entre sus objetivos se encuentran el desarrollar competencias tecnológicas para acceder a fuentes de trabajo emergentes, aumentar la competitividad entre las empresas que tienen en el manejo de la información su principal materia prima y en la generación del conocimiento un capital de transformación económica importante (INTEF, 2017; ISTE, 2018).

De esta forma, los países de la región latinoamericana han elaborado agendas digitales diseñadas para que el sector educativo incorpore en la formación profesional el uso de herramientas tecnológicas con el fin de ofrecer a través de los egresados una respuesta a las demandas laborales identificadas por estos organismos de cooperación internacional con el fin de favorecer la inclusión social, la democratización de la educación y la reducción de la brecha digital (Henriquez et al., 2018).
En este sentido, se ha señalado que existen cuatro razones principales por las que los Estados deben considerar en el diseño de sus políticas educativas el destinar fondos para incorporar las TIC: 1) las demandas laborales de los empleadores que requieren recursos humanos con las competencias digitales necesarias por integrarse a los nuevos modelos productivos, 2) las necesidades sociales en las que impera la urgencia de reducir la brecha digital, 3) los cambios culturales que orientan las relaciones dentro y fuera del aula con el uso de la tecnología, 4) las expectativas pedagógicas en las que las TIC contribuyen en la modernización de las estrategias de aprendizaje (Almerich et al., 2018).

Sin embargo, y a pesar de que se ha impulsado la adquisición de la infraestructura tecnológica en las universidades, pareciera que la práctica docente se encuentra anclada en una era analógica, no en el sentido de que las herramientas digitales estén ausentes en el aula, sino porque las actividades escolares siguen representando modelos de interacción cercanos a la dinámica de la instrucción tradicional debido a que los docentes no se encuentran suficientemente preparados para incorporar las tecnologías en la enseñanza (Avello et al., 2016; Pérez \& Rodríguez, 2016; Romero et al., 2017), incluso se ha afirmado que no han alcanzado las competencias para usar herramientas digitales como un elemento mediador que fortalezca el proceso de enseñanza-aprendizaje (Batane $\&$ Ngwako, 2017; Instefjord \& Munthe, 2017; Passey et al., 2018).

$\mathrm{Al}$ respecto, cabe mencionar que la incorporación de las TIC se ha convertido en una actividad azarosa debido a que son las universidades las que bajo los propios principios administrativos de crecimiento tecnológico se han encargado su decidir qué herramientas digitales son las necesarias para vincular la enseñanza con la sociedad del conocimiento, y escasamente han considerado las necesidades de apropiación tecnológica para cada disciplina profesional y para cada perfil académico (Ilomäki et al., 2016). Esto ha propiciado que los conocimientos para usar con eficiencia las TIC se traduzcan en indicadores generales comúnmente llamados competencias 


\section{Revista Caribeña de Investigación Educativa | 2021, 5(1), 101-115}

digitales, los cuales al ser aplicados han originado prácticas de sustitución de dispositivos análogos por digitales, por ejemplo, se reemplaza el uso del pizarón por un proyector digital, o bien el fotocopiado de un libro por la apertura de un archivo de texto digital (López \& Ramírez, 2016).

El preámbulo descrito indica que en la transferencia de recomendaciones internacionales a las políticas de Estado se están omitiendo las condiciones, tanto del origen de las recomendaciones como de las características de infraestructura tecnológica del país en el que se implementan, con la justificación de que existe una urgencia de que los países reformen sus sistemas educativos para favorecer la relación de la universidad con las demandas laborales del mercado global.

\section{Organismos de cooperación internacional y educación con TIC}

Las orientaciones internacionales vinculadas con la incorporación de las TIC en la educación pueden generar modelos de gobernanza en los que se diluye el poder del Estado para delimitar sus propias políticas, en este sentido, la aparición de esquemas de expansión de la educación a bajo costo mediante la adopción y adaptación de las tecnologías puede propiciar un impacto en la transformación de los sistemas educativos nacionales, sin embargo, el acceso a las TIC aún es una tarea en América Latina (Lugo \& Ithurburu, 2019).

De esta forma, si un país desea legitimar su política de Estado relacionada con la educación, esta debe ajustarse a los términos económicos, tecnológicos y culturales emanados de entes reguladores de cooperación internacional, es decir, debe alinearse a recomendaciones y orientaciones emitidas por organismos que por sus características tienen influencia para: a) desempeñar una importante función discursiva para articular la política de educación superior, b) actuar como redes para construir plataformas políticas para la difusión de propuestas, c) construir una dirección común en la implementación de políticas en la educación superior global (Shahjahan, 2012).
Especialmente, ante el reto de incorporar las tecnologías en la educación a nivel internacional, organismos como la Unesco, la OCDE y la OEI han emitido diversas recomendaciones, algunas desde la década de 1970, que se centran en conceptualizar la importancia de generar competencias digitales en los estudiantes, en la responsabilidad de los gobiernos para promover una enseńanza mediada por las tecnologías y en la urgencia de apropiar el uso de las tecnologías en los docentes.

Aunado a lo anterior, estos mismos organismos han generado iniciativas que tienen la intención de ayudar a los Estados a analizar y evaluar el nivel de apropiación tecnológica mediante la publicación de indicadores y estándares de competencias digitales que han sido ampliamente analizados por diversos autores (Cela et al., 2017; Pettersson, 2017; van Laar et al., 2017; Castańeda et al., 2018), quienes convergen en que la idea de que este tipo de instrumentos tienen la premisa de que pueden servir para diseñar políticas públicas que beneficien el desarrollo de prácticas educativas innovadoras y, con ello, contribuir al desarrollo de la infraestructura digital y de modelos pedagógicos disruptivos.

Sin embargo, estas iniciativas pueden generar tensiones y contradicciones entre el diseño de las políticas públicas y su aplicación en los contextos educativos, sobre todo en aquellos en los que la infraestructura digital es escasa o incluso inexistente. Con el fin de conocer cuáles son los términos en los que se sustenta la incorporación de las TIC desde el enfoque internacional, en los siguientes párrafos se realiza una revisión de las recomendaciones realizadas por los tres organismos.

\subsection{Recomendaciones de la Unesco}

El primer referente del tema de la incorporación de las tecnologías en la educación superior se encuentra en el informe Aprender a ser: La educación del futuro (Faure et al., 1972), en el que se afirma:

[...] la tecnología podría crear condiciones para detonar el surgimiento de un aprendizaje eficaz y rápido mediante las posibilidades de la informática para agilizar la gestión administrativa de la 
enseñanza, el almacenamiento de la información y el uso del "ordenador" para la enseñanza superior de materias complejas ya que se tenía la idea que "el uso del ordenador está llamado también a desempeñar un papel fundamental al servicio de la educación permanente" (p. 199).

En este informe se afirma que incorporar las tecnologías supondría una revolución intelectual desde la que surgirían nuevas habilidades docentes, lo que a su vez daría como resultado una mutación del acto educativo. En el Simposio de Roma (Unesco, 1984) se retoma esta idea, ya que se menciona la importancia de las "nuevas tecnologías": la informática, la telemática, la teleconferencia, la audioconferencia, la mensajería electrónica, los bancos de datos, el magnetoscopio y el videodisco como medios para el aprendizaje que, vinculados con medios como la radio y la televisión, abrirían el camino para cultivar el surgimiento de estrategias educativas así como nuevos modelos para potenciar la masificación de la educación.

Sin embargo, también se afirmaba que para integrar las tecnologías emergentes se requería la mediación de cambios pedagógicos, ya que "para dominarlas mejor es necesario comprender las repercusiones de esos cambios rápidos en la vida individual y colectiva, tanto en el plano social como económico y cultural" (Unesco, 1984, p. 22); posteriormente, en el documento The Introduction of the New Information Technologies in Higher Education (Malitza, 1989), también se aseveraba que los equipos de cómputo estaban transformando la educación superior debido al aumento de las capacidades de almacenamiento, el creciente desarrollo de software y a que "la enseńanza moderna de las disciplinas en la educación superior emplea la metodología en el modelado en el ordenador" (Malitza, 1989, p. 12).

Con estos antecedentes, la Unesco consideró que "la microcomputadora se ha convertido en numerosas sociedades en el símbolo de la modernización de la educación” (Jouët \& Coudray, 1993, p. 49); por otra parte, en distintos informes (Unesco, 1995, 1996, 1998a, 1998b) se mantiene la idea de que las TIC ocupan una posición dominante como un mecanismo transformador de la educación y en las que se deposita la expectativa para lograr cambios importantes en la educación superior, sin embargo, también se manifestó que las TIC no pueden resolverlo todo, ya que primero es necesario eliminar aquellos obstáculos socioeconómicos, culturales y políticos que se oponen al acceso autentico, pertinente y efectivo a la educación.

Posteriormente se publican las Normas Unesco sobre Competencias TIC para Docentes (2008), que tienen como objetivo proponer un marco de referencia para analizar y evaluar el uso de las tecnologías en el escenario educativo, mencionando que disponer de equipos de cómputo en las aulas no siempre es suficiente para garantizar que los docentes tengan las habilidades para guiar u orientar a sus estudiantes para que movilicen saberes digitales, procedimentales y actitudinales que permitan fortalecer sus competencias para responder a las demandas de la sociedad del conocimiento.

En este documento se considera que lo más importante es transitar hacia la realización social de los ciudadanos usuarios de las tecnologías para favorecer su buen desenvolvimiento en la vida social, laboral y profesional, de igual forma se afirma que, a través del uso de las TIC, los estudiantes de un país, los ciudadanos y trabajadores deben desarrollar competencias cada vez más vinculadas con las tecnologías para apoyar el avance económico, social, cultural y ambiental $y$, con ello, tener un mejor nivel de vida.

Posteriormente, la Unesco actualizó su orientación al publicar el Marco de Competencias TIC para Profesores (Unesco, 2011), en el que enfatiza la necesidad de que los sistemas educativos atiendan los objetivos sociales y económicos de los países mediante la formación de los docentes, los cuales no solamente deben tener competencias disciplinares para enseñar a sus estudiantes, sino que deben tener la capacidad de orientarlos para convertirse en los ciudadanos de la sociedad del conocimiento.

Finalmente, en la segunda década de milenio, el organismo replantea el desarrollo de la educación con el uso de las tecnologías (Unesco, 2015) y publica 
el Marco de Competencias y Estándares TIC para Docentes desde la Dimensión Pedagógica (Valencia et al., 2016), que pretende ser un referente para mejorar la calidad educativa a través del uso de las TIC, resaltando que esta propuesta se hace desde un abordaje de los usos educativos de las TIC y con un enfoque pedagógico que se contrapone a la idea original del organismo en el sentido de que la pedagogía debe ajustarse a las novedades tecnológicas.

De esta forma pueden identificarse dos momentos diferentes en las recomendaciones de este organismo (ver figura 1), el primero en el periodo de 1972 a 2009, el cual se centra en ubicar a las TIC como un elemento clave para desarrollar la educación superior y vincularla con las demandas productivas de la sociedad del conocimiento, y el segundo, de 2008 a 2016, en el que se generan marcos para el análisis y la evaluación de las competencias necesarias para que docentes y estudiantes puedan incorporarse a una sociedad cada vez más interconectada.

\section{Figura 1. Recomendaciones de la Unesco respecto del uso de las TIC}

\begin{tabular}{|c|c|}
\hline $1972-2009$ & 2008-2016 \\
\hline $\begin{array}{l}\text { Aprender a ser: La educación } \\
\text { del futuro (Faure et. al., 1972) } \\
\text { The Introduction of the New } \\
\text { Information Technologies in } \\
\text { Higher Education (Malitza, } \\
\text { 1989) } \\
\text { Las Nuevas Tecnologías de } \\
\text { Comunicación: Orientaciones } \\
\text { de la Investigación (Jouët y } \\
\text { Coudray, 1993) } \\
\text { Documento de política para } \\
\text { el cambio y el desarrollo de la } \\
\text { educación superior (UNES- } \\
\text { CO, 1995) } \\
\text { Conferencia Mundial de Edu- } \\
\text { cación (UNESCO, 1996) } \\
\text { From Traditional to Virtual: } \\
\text { The New Informatión Techno- } \\
\text { logies (UNESCO, 1998a) } \\
\text { Declaración Mundial sobre } \\
\text { la Educación Superior en el } \\
\text { Siglo XXI (UNESCO, 1998b) } \\
\text { Conferencia Mundial sobre la } \\
\text { Educación Superior (UNES- } \\
\text { CO, 2009) }\end{array}$ & $\begin{array}{l}\text { Normas UNESCO sobre } \\
\text { Competencias TIC para } \\
\text { Docentes (UNESCO, 2008) } \\
\text { Marco de Competencias } \\
\text { TIC para Profesores } \\
\text { (UNESCO, 2011) } \\
\text { Marco de Competencias y } \\
\text { Estándares TIC para Do- } \\
\text { centes desde la Dimensión } \\
\text { Pedagógica (Valencia, et. } \\
\text { al. 2016) }\end{array}$ \\
\hline $\begin{array}{c}\text { Recomendaciones respecto } \\
\text { a la pertinencia de utilizar } \\
\text { las TIC en la Educación } \\
\text { Superior }\end{array}$ & $\begin{array}{c}\text { Propuestas para utilizar } \\
\text { las TIC en la escuela a } \\
\text { través de la formación de } \\
\text { competencias }\end{array}$ \\
\hline
\end{tabular}

\subsection{Recomendaciones de la OCDE}

La Organización para la Cooperación y Desarrollo Económico (OCDE) es un organismo internacional, multilateral e intergubernamental que realiza estudios periódicos para identificar las problemáticas comunes de la sociedad y fija estándares y recomendaciones que al ser adoptados por sus países miembros les otorga credibilidad ante los principales actores del desarrollo económico internacional (Treviño, 2015).

En sus estatutos, la OCDE no obliga a sus miembros a transformar sus recomendaciones en política pública, sin embargo, los acuerdos tomados en el seno de sus convenciones se reflejan comúnmente en acciones políticas que están alineadas con determinadas iniciativas. En este sentido, en lo que respecta al uso y aprovechamiento de las tecnologías digitales, ha sido reconocida como un factor de cambio en la economía de los países, ya que se tiene un sentido de urgencia para formar capacidades tecnológicas que permitan la incorporación de los ciudadanos a los esquemas laborales mediados por entornos tecnológicos (OCDE, 2010).

El discurso de la OCDE tiene su origen en el vínculo entre la economía, el desarrollo social y la educación (OCDE, 1990), que hace necesario generar estrategias para conducir la aparición de las llamadas "nuevas tecnologías" hacia el desarrollo de la educación superior y las nuevas modalidades de empleo. Dichas estrategias se enuncian en diversos informes como Education and the Economy in a Changing Society (1989) y The OECD Jobs Study: Evidence and Explanations (1994), en los que se declara la necesidad de identificar los cambios y las demandas que se darían en la universidad como consecuencia de la incorporación de las tecnologías en los sectores productivos.

En los años noventa, la OCDE redactó sus informes para enfatizar la relación del concepto de sociedad del conocimiento con la economía, la información, la comunicación, el empleo y el diseño de políticas educativas, afirmando que "el conocimiento es ahora reconocido como el conductor de la productividad y el crecimiento económico, dando lugar a un nuevo 
enfoque del rol de la información, la tecnología y el aprendizaje en el desempeño económico" (OCDE, 1996, p. 3).

La OCDE (2000) también identificó a las tecnologías y las habilidades digitales como un elemento diferenciador para estimular el crecimiento de la economía del aprendizaje debido a la capacidad de estas herramientas para modificar las condiciones en las que se hace el intercambio de información así como de potenciar la agilidad de los procesos educativos de todo tipo, ya que "forzarán un cambio radical en la organización jerárquica, una de las más prominentes características de la modernidad" (p. 57).

Por lo anterior, la OCDE realizó diversas recomendaciones (OCDE, 2000, 2005, 2007) en las que se destaca la prioridad de utilizar activamente el desarrollo tecnológico para modernizar las formas y los recursos para fortalecer la enseñanza y aprendizaje de los estudiantes, haciendo hincapié en el surgimiento y desarrollo de modelos de formación como la educación mixta y a distancia. Estas recomendaciones se aproximaron hacia la masificación de la educación superior, ya que consideraban que el futuro de la incorporación de las TIC se encontraba en la virtualización de los servicios educativos.

Sin embargo, no se consideraban las disparidades de la infraestructura tecnológica predominantes en la región, por ejemplo, en el caso de México, la ANUIES (2000) diseñó el Plan Maestro de Educación Abierta y a Distancia que no fue operativo de forma inmediata debido a la escasa infraestructura tecnológica de las universidades del país. Incluso en el año 2005 en México se contaba con una débil conectividad a internet, mientras que en países como Canadá o Brasil ya existía el desarrollo de cursos virtuales, la masificación de repositorios de objetos y la administración y aplicación de distintos sistemas de gestión de aprendizaje (OCDE, 2005).

Debido a lo anterior, la OCDE rectificó y afirmó que "no existía evidencia acerca de que las TIC pudieran realmente modificar y mejorar las prácticas pedagógicas, ni en lo virtual ni en lo presencial, ni tampoco haber revolucionado el aprendizaje y la enseñanza”
(OCDE, 2007, p. 21), ya que incluso en los países con mayor desarrollo económico "el mayor beneficio de las TIC en la educación superior se dio en los servicios administrativos" (OCDE, 2007, p. 41).

Como consecuencia, la OCDE emite distintas publicaciones (Rychen et al., 1999; Rychen \& Salganik, 2001, 2003) vinculadas con la importancia de la generación de competencias digitales clave para fomentar el bienestar económico, social y para la vida de las personas en un mundo cada vez más conectado. Posteriormente publica el informe Working Paper 21st Century Skills and Competences for New Millennium Learners in OECD Countries (OCDE, 2009), que tiene como objetivo conceptualizar y analizar los efectos de las "nuevas tecnologías digitales" en el desarrollo cognitivo de los estudiantes, especialmente con aquellas relacionadas con las expectativas de desarrollo de calidad educativa.

En este sentido, puede identificarse que las recomendaciones de la OCDE se han desarrollado para lograr tres habilidades: 1) para hacer un buen uso de las diferentes aplicaciones informáticas, 2) para aprender con las tecnologías en las que se combinan actividades cognitivas con habilidades funcionales, 3) aquellas necesarias para interactuar en la sociedad del conocimiento, enmarcadas en dos niveles, el primero relacionado con la generación del conocimiento y el segundo con la transmisión efectiva de la información (Morales, 2018).

\subsection{Recomendaciones de la OEI}

La Organización de Estados Iberoamericanos para la Educación, la Ciencia y la Cultura (OEI) es un organismo internacional intergubernamental creado para articular la cooperación entre sus países miembros en el ámbito educativo; históricamente ha contribuido desde las Declaraciones de las Conferencias Iberoamericanas de Ministros de Educación a impulsar el uso de las tecnologías mediante la ejecución de acciones encaminadas a incorporar estas herramientas en las instituciones formativas.

El discurso de la OEI comienza en 1988, cuando en el marco de la Primera Conferencia, el organismo 
consideró que las TIC deberían incorporarse en los procesos educativos, primero para contribuir en el desarrollo de los procesos de producción, y posteriormente para expandirse en la vida social y cultural de las personas, de esta forma, los ciudadanos estarían mejor preparados para afrontar las problemáticas sociales relacionadas con el acceso a la educación y al empleo (OEI, 1988).

Así, el uso de las TIC para la OEI se centró en fortalecer los espacios audiovisuales mediados por la televisión educativa, y estableció estrategias para robustecer la cooperación entre las universidades y las televisoras de servicio público para formar recursos humanos mediante la transmisión de programas educativos, que sirvió como antecedente para consolidar el espacio audiovisual iberoamericano en el que se privilegió la formación de profesores, la educación para el empleo, la alfabetización, la previsión sanitaria y la conservación de la naturaleza (OEI, 1992b).

En la década de los noventa, la OEI consideró a la educación abierta y a distancia como un componente necesario para acompañar los sistemas de educación permanente y formación continua, ya que se propuso incorporar la experiencia de organizaciones especializadas en esta modalidad educativa para llevar la educación a las comunidades más alejadas de los centros educativos y con ello ampliar la oferta educativa en todos los niveles, poniendo énfasis en esta y en posteriores conferencias en la importancia de la formación del docente para convertirlo en especialista en el uso educativo de las TIC (OEI, 1993, 1995, 1996).

Posteriormente, y con el advenimiento de la sociedad del conocimiento, el organismo afirmó que las "nuevas tecnologías" son elementos posibilitadores para la generación de nuevos paradigmas pedagógicos, así como para la expansión de la oferta educativa en el contexto de la globalización (OEI, 1999), de esta forma, confirmó la idea de que la educación no puede permanecer al margen del avance de las TIC ni de la sociedad del conocimiento (OEI, 2002, 2004), sin embargo, se identificó que existe una brecha digital que predomina en distintas regiones como resultado de la revolución desigual del desarrollo de la infraestructura y de la informática.

Sin embargo, fue en el documento "Metas educativas 2021: La educación que queremos para la generación de los bicentenarios", aprobado en la Declaración de Buenos Aires, que se puso especial énfasis en incorporar las tecnologías en los sistemas educativos nacionales con el objetivo de usarlas como herramientas para lograr una educación de calidad para todos, así como la integración plena de los docentes y los estudiantes a la sociedad del conocimiento (OEI, 2010), de esta forma se identificó que la incorporación de las TIC va más allá de la presencia de herramientas tecnológicas en el espacio escolar, porque se trata también de generar una cultura digital y la adquisición de competencias para el siglo XXI.

Por lo que desde la perspectiva de la OEI se consideran tres dimensiones en las recomendaciones de la incorporación de las tecnologías: 1) las políticas públicas, 2) la escuela, 3) los alumnos, centrando la atención en la dimensión escuela, la cual puede representarse como una matriz de evaluación con cuatro indicadores: 1) disponibilidad de las TIC, 2) organización de la escuela para el uso de las TIC, 3) formulación de los educadores en el uso de las TIC, 4) presencia de las TIC en prácticas pedagógicas.

\section{Implicaciones de las recomendaciones de los organismos internacionales}

Las recomendaciones de la Unesco, la OCDE y la OEI convergen en la necesidad de incorporar las TIC en los sistemas educativos, primordialmente por razones guiadas por el desarrollo económico, así como por la necesidad de vincular la formación universitaria con las demandas de los sectores productivos y la sociedad del conocimiento; lo anterior sugiere que los organismos de cooperación internacional han ejercido el poder de su discurso para hacer valer su influencia como grupo dominante en el plano internacional. 
En este sentido pareciera que los Estados han decidido incorporar estas herramientas sin considerar variables de contexto importantes como la infraestructura tecnológica, el acceso a herramientas digitales, la alfabetización digital, etc. Un ejemplo visible de lo anterior lo podemos observar en la existencia de brechas muy pronunciadas en los niveles de infraestructura tecnológica en los Estados latinoamericanos (CEPAL, 2015b), que se pueden ilustrar, en parte, a través de los indicadores emitidos en el Índice de Desarrollo de las Tecnologías de Información y Comunicación de la Unión Internacional de Telecomunicaciones de Naciones Unidas, los cuales muestran diferencias visibles en el crecimiento de internet, así como en el acceso y utilización de dispositivos digitales (ver Tabla 1), es decir, hay diferencia entre lo que debe ser y lo que se puede hacer con las tecnologías.

Tabla 1. Índice de Desarrollo de las TIC en países latinoamericanos

\begin{tabular}{|c|c|c|c|}
\hline País & Rank 2017 & Rank 2016 & Valor en el índice de desarrollo ${ }^{1}$ \\
\hline Uruguay & 42 & 48 & 7.16 \\
\hline Ecuador & 97 & 101 & 4.84 \\
\hline Nicaragua & 130 & 132 & 3.27 \\
\hline Rep. Dominicana & 106 & 107 & 4.51 \\
\hline Colombia & 84 & 84 & 5.36 \\
\hline Perú & 96 & 97 & 4.85 \\
\hline Costa Rica & 60 & 57 & 6.44 \\
\hline Argentina & 51 & 52 & 6.79 \\
\hline México & 87 & 90 & 5.16 \\
\hline Chile & 56 & 59 & 6.57 \\
\hline Cuba & 137 & 135 & 2.91 \\
\hline
\end{tabular}

Fuente: Elaboración propia a partir de ITU (2018).

Sin embargo, no siempre el acceso a dispositivos se relaciona con la puesta en marcha de estrategias didácticas que signifiquen una innovación de los procesos educativos, principalmente porque los docentes no han desarrollado afinidades tecnológicas (Casillas et al., 2016), es decir, no en todos los países la implementación de las políticas públicas ha permitido que los profesionales de la educación se encuentren

\footnotetext{
${ }^{1}$ El valor se obtiene de la Infraestructura y acceso a las TIC (números de teléfonos fijos cada 100 habitantes, suscriptores a teléfono celulares cada 100 habitantes, ancho de banda de internet respecto de los usuarios de internet, proporción de hogares con computador y porcentaje de hogares con acceso a internet en el domicilio), su uso y la intensidad del mismo (que se mide a través de usuarios de internet cada 100 habitantes, suscriptores de internet de banda ancha fija por cada 100 habitantes y suscriptores de internet de banda ancha móvil por cada 100 habitantes) y capacidades necesarias para hacer un uso efectivo de dicha tecnologia (aproximada a través de la tasa de alfabetización de los adultos, la tasa bruta de matriculación en la educación secundaria y la tasa de matriculación en educación terciaria).
}

capacitados para hacer una transformación digital del proceso de enseńanza-aprendizaje, sino más bien ha provocado que los docentes utilicen las tecnologías para acompańar pedagogías tradicionalmente dominantes como la exposición oral de la clase (Howard \& Thompson, 2016; Claro et al., 2018; Salado et al., 2019).

Sin embargo, se debe mencionar que Cuba, con uno de los índices de desarrollo de las TIC más bajos de la región, ha logrado tener el mayor porcentaje de profesores capacitados en el uso de las tecnologías, en parte debido a que la incorporación de estas herramientas en los procesos de enseñanza se ha desarrollado desde la perspectiva de que lo más importante es trabajar en el desarrollo de las habilidades docentes para diseñar estrategias didácticas, y posteriormente en la capacitación en el uso de herramientas tecnológicas (Carnoy et al., 2010). 
Por lo anterior, se puede sugerir que desde los organismos de cooperación internacional se deben generar marcos de referencia con el uso de las TIC eminentemente pedagógicos, en los que el principal factor de éxito en la incorporación tecnológica sea la generación de estrategias contextualizadas para cada nivel educativo y, en el caso de la educación superior, para cada área disciplinar; en este orden de ideas, la incorporación de las tecnologías debería estar condicionada por la funcionalidad que los recursos digitales aportan al momento de resolver problemáticas específicas y con ello agreguen un valor significativo al fortalecimiento de los métodos inherentes a cada área de conocimiento.

Finalmente, el rol del docente en el escenario de la integración de las TIC no debe limitarse solamente a la capacitación para el uso de determinadas herramientas digitales, sino que debe considerar la amplitud de las dimensiones de la práctica en el aula, en el sentido de que esta actividad es multivariada, por ello, las orientaciones de los organismos internacionales deben centrarse en generar habilidades para crear recursos digitales, para enriquecer ambientes de aprendizaje presenciales con herramientas virtuales, así como modernizar las prácticas pedagógicas, ya que no son las tecnologías las que cambian los procesos de enseńanza aprendizaje, sino los modelos pedagógicos.

\section{Conclusiones}

La implementación de las políticas públicas incide a gran escala en las estrategias de incorporación de las tecnologías en las instituciones educativas (Bottino, 2020); en la actualidad, la penetración de la infraestructura tecnológica, la masificación de la conectividad a internet, así como el acceso a los dispositivos digitales en las universidades ha provocado que exista una percepción de éxito en cuanto al desarrollo de las habilidades que permiten a los docentes utilizar las tecnologías y la información de forma adecuada para concretar objetivos educacionales (Hargittai \& Dobransky, 2017; Sánchez et al., 2017; Hanbidge et al., 2018; Walton et al., 2018). Sin embargo, habría que preguntarse si las orientaciones que emanan de los organismos de cooperación internacional están impactando solamente en el aumento de la infraestructura tecnológica o si también están contribuyendo a generar saberes relacionados con la construcción de estrategias pedagógicas que hagan que los docentes enseñen y aprendan mediante las tecnologías.

La respuesta depende de las interpretaciones que la propia universidad interioriza acerca de las recomendaciones de política pública, así como de sus pretensiones de posicionamiento local, nacional e internacional respecto a indicadores de integración de infraestructura tecnológica y formación de recursos humanos que son usuarios de las TIC. Sin embargo, independientemente de las muy variadas interpretaciones, es urgente incluir en las agendas nacionales planes concretos para lograr una integración de las tecnologías de forma ordenada y contextualizada que signifique no solo el uso de las herramientas electrónicas de moda, sino un cambio en los paradigmas educativos.

Por consiguiente, en el tema de la transformación de la práctica educativa mediada por el uso de las tecnologías debe superarse la idea de que contar con computadoras, software y conectividad a internet es suficiente para que exista un surgimiento de nuevas pedagogías (Rovira \& Stumpo, 2015). En este sentido, integrar la tecnología debe entenderse como una realineación del sistema educativo existente en el que el docente continúa siendo un expositor de información, hacia una experiencia más centrada en el estudiante y mediada por dispositivos digitales (McGarr \& Johnston, 2019); lo anterior sugiere que se debe abandonar la idea de que incorporar es simplemente colocar dispositivos en las aulas, sino que también es formar intencionadamente y con apego a las intenciones del currículum escolar a los docentes, es medir los cambios en las estrategias de enseñanza y el impacto en la adquisición y transformación de los aprendizajes (Pereira et al., 2017).

Para concluir, se debe reconocer que la incorporación de las TIC en la universidad es una realidad que ha sido abordada con detenimiento por diversos organismos internacionales, los cuales han determinado que 
el uso de estas herramientas es esencial para consolidar el fortalecimiento social y económico de las naciones. En ese sentido, es prioritario aprender a utilizarlas y colocarlas en el pensamiento del docente y el estudiante como un elemento que le permitirá tener un mayor éxito para encontrar mejores oportunidades de desarrollo intelectual y laboral (Taylor, 2017).

Sin embargo, es necesario reconocer que el uso instrumental, poco intencionado y sin propósitos educativos de las TIC puede originar una simulación del desarrollo de la incorporación digital en los procesos de enseñanza y aprendizaje, por lo que resulta necesario asumir una postura crítica acerca de las interpretaciones de cómo deben implementarse las tecnologías en las aulas y fuera de ellas (van de Oudeweetering \& Voogt, 2018). Por otra parte, el uso de las TIC no solamente debe centrarse en la formación de profesionales para satisfacer las demandas del mercado laboral, sino que también debe extenderse para formar ciudadanos digitales y afines con el desarrollo socioeconómico sostenible y grupal. A modo de cierre, vale plantearse la siguiente pregunta: ¿La transformación de la educación superior puede lograrse sin incorporar las TIC en el aula? La respuesta, más allá de demostraciones empíricas, requiere de un análisis cualitativo de la práctica docente con el uso y sin el uso de las TIC, así como de la observación del impacto del uso de estas herramientas en la enseńanza y el aprendizaje.

\section{Referencias}

Almerich, G., Díaz, I., Cebrián, S., \& Suárez, J. (2018). Estructura dimensional de las competencias del siglo XXI en alumnado universitario de educación. RELIEVE, 24(1). http://doi.org/10.7203/ relieve.24.1.12548

ANUIES (2000). Plan Maestro de Educación Superior y Abierta a Distancia. Lineas estratégicas para su desarrollo. (Documento aprobado en lo general por la XXXI Asamblea General Ordinaria en su sesión del 16 de octubre de 2000 en la Universidad Autónoma de Nuevo León). https://r.issu.edu.do/l?l=767cWk

Arellano, W. (2014). Las politicas de telecomunicaciones y TIC. En R. Amador-Bautista (Ed.), Educación y tecnologías de la información y la comunicación. Paradigmas teóricos de la investigación (pp. 95-132). México: UNAM.

Avello, R., López, R., \& Alpizar, R. (2016). Sistema de formación continua en alfabetización digital para los docentes de las escuelas de hotelería y turismo cubanas. RED. Revista de Educación a Distancia, 49. http://dx.doi.org/10.6018/red/49/7

Batane, T., \& Ngwako, A. (2017). Technology use by pre-service teachers during teaching practice: Are new teachers embracing technology right away in their first teaching experience? Australasian Journal of Educational Technology, 33(1), 58-61. https://doi.org/10.14742/ajet.2299

Bottino, R. (2020). Schools and the digital challenge: Evolution and perspectives. Education and Information Technologies, 25(3), 2241-2259. http://dx.doi.org/10.1007/s10639-019-10061-x

Cabero, J., Romero, R., Barroso, J., \& Palacios, A. (2020). Marcos de Competencias Digitales Docentes y su adecuación al profesorado universitario y no universitario. Revista Caribeña de Investigación Educativa (RECIE), 4(2), 137-158. https://doi.org/10.32541/recie.2020.v4i2. pp137-158

Carnoy, M., Gove, A., \& Marshal, J. (2010). La ventaja académica de Cuba. ¿Por qué los estudiantes cubanos rinden más? México: Fondo de Cultura Económica.

Casillas, M., Ramírez, A., Carvajal, M., \& Valencia, K. (2016). La integración de México a la sociedad de la información. En C. E. Téllez (Ed.), Derecho y TIC. Vertientes actuales. México: Infotec.

Castañeda, L., Esteve, F., \& Adell, J. (2018). ¿Por qué es necesario repensar la competencia docente para el mundo digital? RED. Revista de Educación a Distancia, 56, 2-20. https://doi.org/10.6018/red/56/6

Cela, J. M., Esteve, V., Esteve, F., González, J., \& Gisbert, M. (2017). El docente en la sociedad digital: una propuesta basada en la pedagogía transformativa y en la tecnología avanzada. Profesorado. Revista de 
Currículum y Formación de Profesorado, 21(1), 403422. https://r.issu.edu.do/l?1=76875e

CEPAL (2015b). La nueva revolución digital. De la Internet del consumo al internet de la producción. Santiago de Chile: CEPAL.

Claro, M., Álvaro, T., Cabello, E., San Martín, D., Valenzuela, S., \& Jara, I. (2018). Teaching in a Digital Environment (Tide): Defining and measuring teachers' capacity to develop students digital information and communication skills. Computers \& Education, 121, 162-174.

https://doi.org/10.1016/j.compedu.2018.03.001

Faure, E., Herrera, F., Kaddoura, A., Lopes, H., Petrovski, A., Rahnema, M., \& Champion F. (1972). Aprender a ser. La educación del futuro. Madrid: Alianza Editorial.

Fuentes, A., López, J. \& Pozo, S. (2019). Análisis de la Competencia Digital Docente: Factor Clave en el Desempeño de Pedagogías Activas con Realidad Aumentada. REICE. Revista Iberoamericana sobre Calidad, Eficacia y Cambio en Educación, 17(2), 27-42. https://doi.org/10.15366/reice2019.17.2.002

García, A., \& Ramírez, M. (2019). Systematic Mapping of Scientific Production on Open Innovation (2015-2018): Opportunities for Sustainable Training Environments. Sustainability, 11(6). http://doi.org/10.3390/su11061781

Giraldo, D., \& Báquiro, J. (2020). Appropriation of ICT in the educational field: Approach to public policy in Colombia years 2000-2019. Digital Education Review, (37), 109-129. http://doi.org/10.1344/ DER.2020.37.109-129

Hanbidge, A., Tin, T., \& Sanderson, N. (2018). Information literacy skills on the go: Mobile learning innovation. Journal of Information Literacy, 12(1), 118-136. http://dx.doi.org/10.11645/12.1.2322

Hargittai, E., \& Dobransky, K. (2017). Old dogs, new clicks: Digital inequality in skills and uses among older adults. Canadian Journal of Communication, 42(2), 195-212.

http://dx.doi.org/10.22230/cjc.2017v42n2a3176
Henriquez, C., Gisbert, M., \& Fernández, I. (2018). La evaluación de la competencia digital de los estudiantes: una revisión al caso latinoamericano. Chasqui. Revista Latinoamericana de Comunicación, 137, 93-112. https://doi.org/10.16921/chasqui.v0i137.3511

Howard, S., \& Thompson, K. (2016). Seeing the System: Dynamics and complexity of Technology integration in secondary schools. Education and Information Technologies, 21(6), 1-18.

https://doi.org/10.1007/s10639-015-9424-2

Ilomäki, L., Paavola, S., Lakkala, M., \& Kantosalo, A. (2016). Digital competence. An emergent boundary concept for policy and educational research. Education and Information Technologies, 21(3), 655679. https://doi.org/10.1007/s10639-014-9346-4)

Instefjord, E., \& Munthe, E. (2017). Educating digitally competent teachers: A study of integration of professional digital competence in teacher education. Teaching and Teacher Education, 67, 37-45. https://doi.org/10.1016/j.tate.2017.05.016

INTEF (2017). Marco Común de Competencia Digital Docente. Octubre 2017. Madrid: Instituto Nacional de Tecnologías Educativas y Formación del Profesorado.

ISTE (2018). Crosswalk: Future Ready Librarians Framework and ISTE Standards for Educators. Washington D. C.: International Association for Technology in Education.

ITU (2018). ICT Development Index 2017. https://r. issu.edu.do/l?1=769DCu

Jouët, J., \& Coudray, S. (1993). Las nuevas tecnologias de comunicación: orientaciones de la investigación. París: Unesco.

Lázaro, J., Gisbert, M., \& Silva, J. (2018). Una rúbrica para evaluar la competencia digital del profesor universitario en el contexto latinoamericano. EDUTEC, Revista Electrónica de Tecnología Educativa, 63. http://dx.doi.org/10.21556/edutec.2018.63.1091

López, R., \& Ramírez, A. (2016). Políticas de equipamiento tecnológico en Educación. Debate Universitario, 
Incorporación de las TIC en la educación. Recomendaciones de organismos de cooperación...

5(9), 53-67.

https://r.issu.edu.do/l?1=770rSS

Lugo, M. T., \& Ithurburu, V. (2019). Políticas digitales en América Latina. Tecnologías para fortalecer la educación de calidad. Revista Iberoamericana de Educación, 79(1), 11-31. https://doi.org/10.35362/rie7913398

Malitza, M. (1989). The introduction of the New Information Technologies in Higher Education. París: Unesco.

McGarr, O., \& Johnston, K. (2019). Exploring the Evolution of Educational Technology Policy in Ireland: From Catching-Up to Pedagogical Maturity. Educational Policy, 33(7), 635-643. https://doi.org/10.1177/0895904819843597

Morales, A. (2018). TIC, disciplinas académicas $y$ profesores universitarios. Veracruz: Universidad Veracruzana.

OCDE (1990). Las nuevas tecnologías en la década de los noventa. Una estrategia socioeconómica. Ministerio de Trabajo y Seguridad Social: Madrid

OCDE (1994). The OECD Jobs Study: Evidence and Explanations. París: OCDE.

OCDE (1996). The Knowledge Based Economy. París: OCDE.

OCDE (2000). Knowledge Managment in the Learning Society. París: OCDE.

OCDE (2005). E-learning in Tertiary Education. Where do we stand? París: OCDE.

OCDE (2007). Giving Knowledge for Free. París: OCDE.

OCDE (2009). Working Paper 21st Century Skills and Competences for New Millennium Learners in OECD Countries (EDU Working paper no. 41). París: OCDE.

OCDE (2010). Perspectivas de las tecnologias de la información de la OCDE. París: OCDE.

OEI (1988). Primera Conferencia Iberoamericana de Educación. La Habana, Cuba.
OEI (1992b). III Conferencia Iberoamericana de Educación. Santa Fe de Bogotá, Colombia.

OEI (1993). IV Conferencia Iberoamericana de Educación. Salvador, Bahía, Brasil.

OEI (1995). V Conferencia Iberoamericana de Educación. Buenos Aires, Argentina.

OEI (1996). VII Conferencia Iberoamericana de Educación. Mérida, Venezuela.

OEI (1999). IX Conferencia Iberoamericana de Educación. La Habana, Cuba.

OEI (2002). XII Conferencia Iberoamericana de Educación. Santo Domingo, República Dominicana.

OEI (2004). XIV Conferencia Iberoamericana de Educación. San José, Costa Rica.

OEI (2010). XX Conferencia Iberoamericana de Educación. Buenos Aires, Argentina.

Passey, D., Shonfeld, M., Appleby, L., Judge, M., Saito, T., \& Smits, A. (2018). Digital agency: Empowering equity in and through education. Technology, Knowledge and Learning, 23(3), 425-439. https://doi.org/10.1007/s10758-018-9384-x

Pereira, L., Jorge, A., \& Brites, M. (2017). Media education competitions: An efficient strategy for digital literacies? Italian Journal of Sociology of Education, 9(1), 77-92.

http://dx.doi.org/10.14658/pupj-ijse-2017-15

Pérez, A., \& Rodríguez, M. J. (2016). Evaluación de las competencias digitales autopercibidas del profesorado de educación primaria en Castilla y León (España). Revista de Investigación Educativa, 34(2), 399-415. https://doi.org/10.6018/rie.34.2.215121

Pettersson, F. (2017). On the issues of digital competence in educational contexts - a review of literature. Education and Information Technologies, (23), 10051021. https://doi.org/10.1007/s10639-017-9649-3

Romero, M., Castejón, F., López, V., \& Fraile A. (2017). Evaluación formativa, competencias comunicativas y TIC en la formación del profesorado. Comunicar: Revista Cientifica Iberoame- 
ricana de Comunicación y Educación, 25(52), 73-82. https://r.issu.edu.do/l?l=771fL9

Rovira, S., \& Stumpo, G. (2015). Entre mitos y realidades: TIC, politicas públicas y desarrollo productivo en América Latina. Santiago de Chile: CEPAL.

Rychen, D., \& Salganik, L. (2001). Defining and selecting key competencies. París: OCDE.

Rychen, D., \& Salganik, H. (2003). Key competencies for a successful life and a well-functioning society. París: OCDE.

Rychen, D., Salganik, Moser, L., \& Konstant, J. (1999). Projects on competencies in the OECD context: Analysis of theoretical and conceptual foundations. París: OCDE.

Salado, L., Amavisca, S., Richart, R., \& Rodríguez, R. (2019). Alfabetización digital de estudiantes universitarios en las modalidades presencial y virtual. Revista Electrónica de Investigación e Innovación Educativa, 5(1), 30-47. https://cutt.ly/EyHGWoH

Sánchez, M., De Frutos, B., \& Vázquez, T. (2017). Parent's influence on acquiring critical internet skills. Comunicar, 25(53), 103-111.

http://dx.doi.org/10.3916/C53-2017-10

Shahjahan, R. (2012). The roles of international organizations in globalizing Higher Education Policy. En J. Smart. \& M. Paulsen (Eds.), Higher Education: Handbook of theory and research. Memphis: Springer.

Taylor, A. (2017). Social work and digitalisation: bridging the knowledge gaps. Social Work Education, 36(8), 869-879.

http://dx.doi.org/10.1080/02615479.2017.1361924

Treviño, E. (2015). La educación superior y el advenimiento de la sociedad del conocimiento. México: ANUIES.

Unesco (1984). Simposio sobre el impacto cultural, social y económico de las nuevas tecnologías de la comunicación, Roma, 12-16 de diciembre de 1983. Roma; Instituto de la Enciclopedia Italiana.
Unesco (1995). Documento de politica para el cambio y el desarrollo de la educación superior. París: Unesco.

Unesco (1996). Conferencia Internacional de Educación. Ginebra: Unesco.

Unesco (1998a). From Traditional to Virtual: The New Information Technologies. París: Unesco.

Unesco (1998b). Conferencia Mundial sobre la Educación Superior. La educación superior en el siglo XXI: Visión y acción. Tomo I. París: Unesco.

Unesco (2008). Estándares de Competencia en TIC para Docentes. Londres: Unesco.

Unesco (2011). UNESCO ICT Competency Framework for Teachers. Paris: Unesco.

https://r.issu.edu.do/l?1=772AAw

Unesco (2015). Replantear la educación: ¿Hacia un bien común mundial? París: Organización de las Naciones Unidas para la Educación, la Ciencia y la Cultura.

Valencia, T., Serna, A., Ochoa, S., Caicedo, A., Montes, J., \& Chávez, J. (2016). Competencias y estándares TIC desde la dimensión pedagógica: Una perspectiva desde los niveles de apropiación de las TIC en la práctica educativa docente. Cali: Pontificia Universidad Javeriana.

van de Oudeweetering, K., \& Voogt, J. (2018). Teachers' conceptualization and enactment of twenty-first century competences: exploring dimensions for new curricula. Curriculum Journal, 29(1), 116-133.

http://dx.doi.org/10.1080/09585176.2017.1369136

van Dijk, T. (2009). Análisis del discurso. Discurso y poder. Barcelona: Gedisa.

van Laar, E., van Deursen, A. J., van Dijk, J. A., \& de Haan, J. (2017). The relation between 21st-century skills and digital skills: A systematic literature review. Computers in Human Behavior, 72, 577-588. https://doi.org/10.1016/j.chb.2017.03.010

Walton, G., Pickard, A., \& Dodd, L. (2018). Information discernment, mis-information and pro-active scepticism. Journal of Librarianship and 
Incorporación de las TIC en la educación. Recomendaciones de organismos de cooperación...

Information Science, 50(3), 296-309.

http://dx.doi.org/10.1177/0961000618769980
WEF (2016). The Future of Jobs Employment, Skills and Workforce Strategy for the Fourth Industrial Revolution. Geneva: WEF. https://goo.gl/yhKr9j

\section{CÓMO CITAR:}

Incorporación de las TIC en la Educación. Recomendaciones de organismos de cooperación internacional 1972-2018. RECIE. Revista Caribeña de Investigación Educativa, 5(1), 101-115. https://doi.org/10.32541/recie.2021.v5i1.pp101-115 